\title{
Simple and Exact Closed-Form Expression for Determination of a Penetrated Ray Path in a Ray Tracing
}

\author{
Hyun Wook Moon, ${ }^{1}$ Woojoong Kim, ${ }^{1}$ Sewoong Kwon, ${ }^{2}$ \\ Jaeheung Kim, ${ }^{3}$ and Young Joong Yoon ${ }^{1}$ \\ ${ }^{1}$ Department of Electric and Electronic Engineering, Yonsei University, Seoul 120-749, Republic of Korea \\ ${ }^{2}$ ISR R\&D Laboratory LIG Nex1, 207, Mabuk-ro, Giheung-gu, Yongin-si, Gyeonggi-do 446-798, Republic of Korea \\ ${ }^{3}$ Yonsei Institute of Convergence Technology, Yonsei University, Incheon 406-840, Republic of Korea \\ Correspondence should be addressed to Young Joong Yoon; yjyoon@yonsei.ac.kr
}

Received 29 July 2013; Accepted 13 November 2013

Academic Editor: Felipe Cátedra

Copyright (C) 2013 Hyun Wook Moon et al. This is an open access article distributed under the Creative Commons Attribution License, which permits unrestricted use, distribution, and reproduction in any medium, provided the original work is properly cited.

\begin{abstract}
A simple and exact closed-form equation to determine a penetrated ray path in a ray tracing is proposed for an accurate channel prediction in indoor environments. Whereas the penetrated ray path in a conventional ray tracing is treated as a straight line without refraction, the proposed method is able to consider refraction through the wall in the penetrated ray path. Hence, it improves the accuracy in ray tracing simulation. To verify the validation of the proposed method, the simulated results of conventional method, approximate method, and proposed method are compared with the measured results. The comparison shows that the proposed method is in better agreement with the measured results than the conventional method and approximate method, especially in high frequency bands.
\end{abstract}

\section{Introduction}

In recent years, a lot of indoor wireless applications have been proposed and researched to satisfy various demands such as high data rates, low power consumption [1]. In addition, the operating frequencies of these indoor applications are going up to millimeter-wave wireless personal area networks (WPAN) due to a number of merits and the lack of available frequency bands [1-3]. In order to deploy these applications successfully, exact prediction and detailed understanding of the wave propagation characteristics in indoor environments are necessary. Therefore, there have been many attempts to model indoor radio channel as accurately as possible by using ray tracing $[4,5]$.

The ray tracing technique which is based on geometrical optics (GO) and uniform theory of diffraction (UTD) is capable of calculating an electromagnetic field at a receiving point by adding multipath waves coherently. These multipath waves with different amplitudes and phases depending on wave propagation mechanisms cause deep power fading and/or intersymbol interference (ISI) [6]. Hence, more accurate calculations of amplitude and phase for each multipath wave in ray tracing are very crucial to predict indoor radio channel exactly.

In indoor environments, the penetration through the building wall is one of the important wave propagation mechanisms. In conventional ray tracing, the building wall has been treated as a semi-infinite half-space, and then the electric field for the penetrated ray path is calculated with the Fresnel transmission coefficients because finding the exact penetrated ray path in ray tracing is complex and time-consuming [7-9]. This conventional method makes the assumed penetrated ray path $\left(L^{\prime}\right)$ through the wall as an incorrect straight line although the actual penetrated ray path $(L)$ is refracted through a building wall due to difference of refraction indices between the air and the wall and finite thickness of wall as shown Figure 1. Thus, the path error for the penetrated ray path is caused by incorrect two factors: an inaccurate incident angle $\left(\theta_{i}^{\prime}\right)$, which leads to an error for transmission coefficient, and incorrect total path length from the transmitting point to the receiving point, which leads to a phase error that is proportional to the frequency. As a result, 


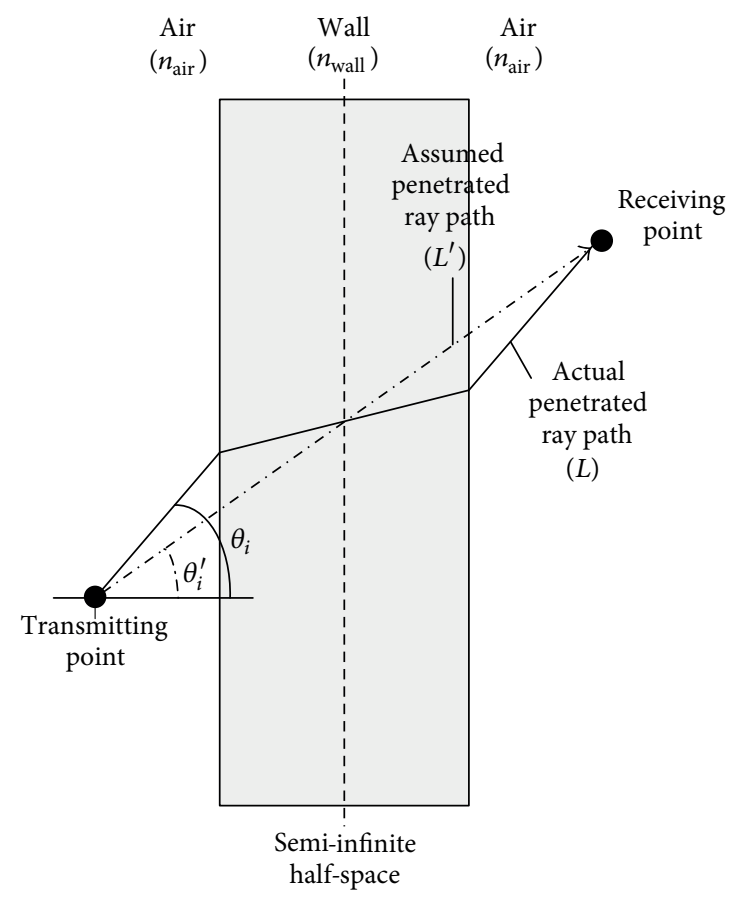

$L^{\prime}$ : Assumed total path length $\theta_{i}^{\prime}$ : Assumed incident angle $L$ : Actual total path length $\theta_{i}$ : Actual incident angle

FIGURE 1: Comparison between an assumed ray path in conventional ray tracing and an actual ray path for penetration through the wall.

this path error can cause an inaccuracy in calculating the fading effect when the multipath waves are superposed after the building wall in indoor environments.

To overcome this path error within the ray tracing framework, a few studies were carried out. In $[10,11]$, it was demonstrated that the path error can be reduced when the number of source rays is increased during shooting and bouncing ray tracing (SBR). However, this approach is still not accurate enough and requires increasing simulation time. In [12], an approximate method for the image theory (IT) to determine the penetrated ray path with relatively small error was proposed. Although the error in this method is negligibly small in low frequency bands, it can cause significant phase error in high frequency bands because the phase error is growing proportionally to frequency. Therefore, this method could result in more error in the ray tracing calculation at a millimeter-wave communication environment. Moreover, the approximate method has some complex procedures since it is not given in a closed-form expression.

In this paper, a closed-form equation to determine the penetrated ray path through the wall with finite-thickness in the ray tracing is proposed. This proposed equation is not only an exact solution for the penetrating ray path because it is derived from Fermat's principle [13], but also simple because it is provided in a closed-form without any complex procedure. To validate the proposed method, fading characteristics at $700 \mathrm{MHz}$ and $7 \mathrm{GHz}$ are measured and then compared with simulation results of ray tracing.

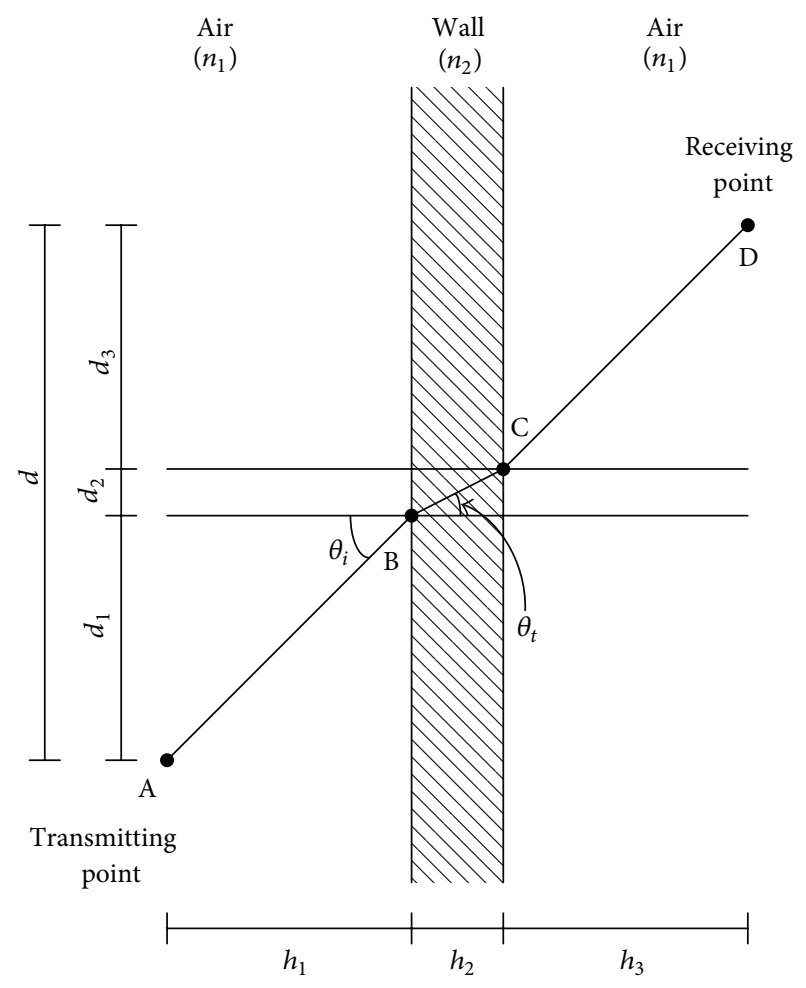

FIGURE 2: Geometrical representation of penetrating ray path through the wall.

The paper is organized as follows. The proposed method is described in Section 2 and an experimental setup to measure the fading characteristics in indoor environments is described in Section 3. The results in terms of fading effect are presented and discussed in Section 4 and conclusions are given in Section 5.

\section{A Closed-Form Expression of the Penetrated Ray Path}

Figure 1 represents an assumed ray path in conventional ray tracing and an actual ray path for penetration through the wall. As mentioned before, since the wall is assumed to be a semi-infinite half-space in conventional ray tracing, the assumed penetrated ray path has two differences compared to the actual penetrated ray path: the incident angle $\left(\theta_{i}\right)$ and the total path length $(L)$. These differences lead to an error in electromagnetic field computations. In order to correct the error and calculate rapidly, a simple and exact closed-form equation to determine the penetrating ray path is derived from Fermat's principle.

Figure 2 represents a penetrating ray path through the wall. During ray tracing, the following information is given from geometric parameters: the locations of the transmitting/receiving points, the thickness of the wall, and the electromagnetic properties of air and the wall. Hence, refractive indices $n_{1}$ and $n_{2}$ and lengths $h_{1}, h_{2}, h_{3}$, and $d$ are known parameters. For a given situation, the goal is to determine the parameters $d_{1}, d_{2}$, and $d_{3}$. 
According to Fermat's principle, the derivative of the optical path length from the transmitting point $(A)$ to the receiving point (D) must be zero to have the shortest path [13]. Using the geometry in Figure 2, the optical total path length (l) for the penetrating ray path, whose $d_{2}$ and $d_{3}$ are substituted by $d_{1}$, is expressed as

$$
\begin{aligned}
l & =n_{1} \overline{A B}+n_{2} \overline{B C}+n_{1} \overline{C D} \\
& =n_{1} \sqrt{h_{1}^{2}+d_{1}^{2}}+n_{2} \sqrt{h_{2}^{2}+d_{2}^{2}}+n_{1} \sqrt{h_{3}^{2}+d_{3}^{2}} \\
& =n_{1} h^{\prime} \sqrt{h_{1}^{2}+d_{1}^{2}}+n_{2} \sqrt{h_{2}^{2}+\left(d-h^{\prime} d_{1}\right)^{2}},
\end{aligned}
$$

where $h^{\prime}=1+h_{3} / h_{1}$. To satisfy Fermat's principle, the derivative of the optical path length $\left(\delta l / \delta d_{1}\right)$ should be zero to have the shortest path. Thus, from this condition, a fourthorder equation which agrees with Snell's law is obtained, as shown in the following equation:

$$
n_{1} \sin \theta_{i}=\frac{n_{1} d_{1}}{\sqrt{h_{1}^{2}+d_{1}^{2}}}=\frac{n_{2}\left(d-h^{\prime} d_{1}\right)}{\sqrt{h_{2}^{2}+\left(d-h^{\prime} d_{1}\right)^{2}}} .
$$

Then, four possible solutions for $d_{1}$ from (2) can be expressed, as shown in the following equation:

$$
\begin{aligned}
& d_{11}=0.5\left(\frac{d}{h^{\prime}}-\sqrt{2 X_{1}-X_{2}+X_{3}}-\sqrt{X_{1}+X_{2}}\right), \\
& d_{12}=0.5\left(\frac{d}{h^{\prime}}+\sqrt{2 X_{1}-X_{2}+X_{3}}-\sqrt{X_{1}+X_{2}}\right), \\
& d_{13}=0.5\left(\frac{d}{h^{\prime}}-\sqrt{2 X_{1}-X_{2}+X_{3}}+\sqrt{X_{1}+X_{2}}\right), \\
& d_{14}=0.5\left(\frac{d}{h^{\prime}}+\sqrt{2 X_{1}-X_{2}+X_{3}}+\sqrt{X_{1}+X_{2}}\right),
\end{aligned}
$$

where

$$
\begin{gathered}
X_{1}=\frac{1}{h^{\prime 2}}\left(d^{2}-\frac{2 Y_{1}}{3 Y_{2}}\right) \\
X_{2}=\frac{1}{3 Y_{2} h^{\prime 2}}\left(Y_{1}^{2}\left(\frac{Y_{3}+\sqrt{Y_{4}}}{2}\right)^{-1 / 3}+\left(\frac{Y_{3}+\sqrt{Y_{4}}}{2}\right)^{1 / 3}\right), \\
X_{3}=\frac{\left(2 d / h^{\prime}\right)\left(Y_{1} / Y_{2} h^{\prime 2}-d^{2} / h^{\prime 2}+2 h_{1}^{2} / Y_{2}\right)}{\sqrt{X_{1}+X_{2}}} \\
Y_{1}=\left(n_{12}^{2}-1\right) d^{2}-h_{1}^{2} h^{\prime 2}+h_{2}^{2} n_{12}^{2} \\
Y_{2}=n_{12}^{2}-1 \\
Y_{3}=2 Y_{1}^{3}+108 Y_{2} d^{2} h_{1}^{2} h_{2}^{2} h^{\prime 2} n_{12}^{2} \\
Y_{4}=Y_{3}^{2}-4 Y_{1}^{6}, \\
n_{12}=\frac{n_{1}}{n_{2}} .
\end{gathered}
$$

Among the above solutions, $d_{12}$ is a unique solution because $d_{1}$ must have a real value in the range $[0, d]$. When the value of $d_{1}$ is fixed, $d_{2}$ and $d_{3}$ can be obtained using the relation between them by Snell's law, and the penetrating ray path is finally calculated by the ray tracing. This result is not only an exact solution for the penetrating ray path because it is derived from Fermat's principle, but also simple because it is provided in closed-form without any complex procedure.

\section{Experimental Setup}

To validate the proposed method, an experiment was performed at an empty office room on the sixth floor of the engineering building at Yonsei University. As shown in Figure 3, the size of the office room was $8.00 \mathrm{~m} \times 6.02 \mathrm{~m}$. It was composed of concrete walls with $20 \mathrm{~cm}$ thickness, a window, and a metal door.

To generate the fading including the penetrating ray path, the transmitter $(\mathrm{Tx})$ was placed in the middle of a corridor outside the office room and the receiver $(\mathrm{Rx})$ was placed in the office room and moved along the paths (1-8), which are $5 \mathrm{~m}$ in length and equally spaced at $50 \mathrm{~cm}$ intervals. The metal door was open resulting in reflection. Hence, the reflecting ray from metal door and various multi-path waves after penetration through the wall were superposed and caused the fading.

Figure 4 shows the measurement system to measure the received signal strength. At the transmitter, the continuous wave $(\mathrm{CW})$ signal was generated from a signal generator (Anritsu MG3695A) and was radiated with $30 \mathrm{dBm}$ transmitting power through a $30 \mathrm{~dB}$ gain amplifier and a reference dipole antenna at $1 \mathrm{~m}$ height. Then at the receiver, the signal was received through an identical reference dipole antenna at $1 \mathrm{~m}$ height and measured by a spectrum analyzer (Agilent 8566B) at the center frequency. This measured received signal strength was stored in a notebook PC through a General Purpose Interface Bus (GPIB) connection. During the measurement, it is very important that the received signal is measured at an exact point in the moving path of the receiver. Hence, four rectangular conductors were mounted on the wheel with equal spacing and a magnetic sensor was also mounted beside the wheel. This magnetic sensor generated trigger signal when it detected the conductors. Thus, the trigger signal which was generated with a regular time interval made the spectrum analyzer operate at the exact point. Since the diameter of the wheel was $7 \mathrm{~cm}$, the spacing between the conductors was about $5.5 \mathrm{~cm}$ and total number of samples in each path was 91 . In order to obtain the fading characteristics, the measurements at each path were repeated ten times and final received signal strength was calculated by their average value.

\section{Fading Effect Comparison}

Figure 5 shows measured received signal strength with unit $\mathrm{dBm}$ at $700 \mathrm{MHz}$ and $7 \mathrm{GHz}$. The fading of the multi-path waves is shown and the fading effect is more severe and frequent in higher frequency bands because the phase for 


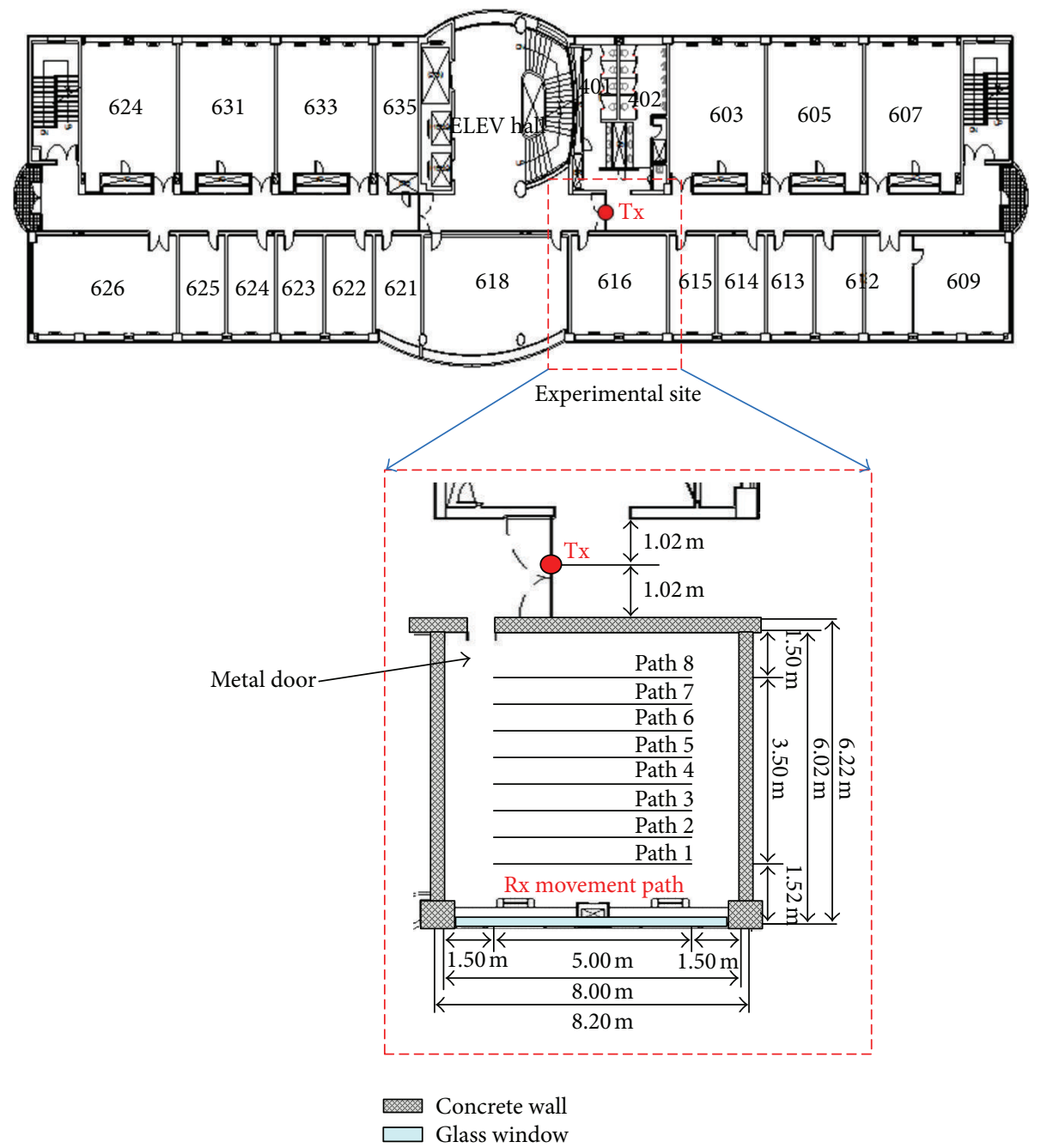

Figure 3: Experimental site.

Reference dipole antenna

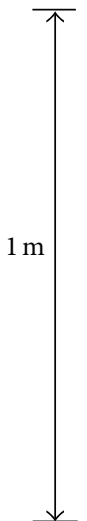

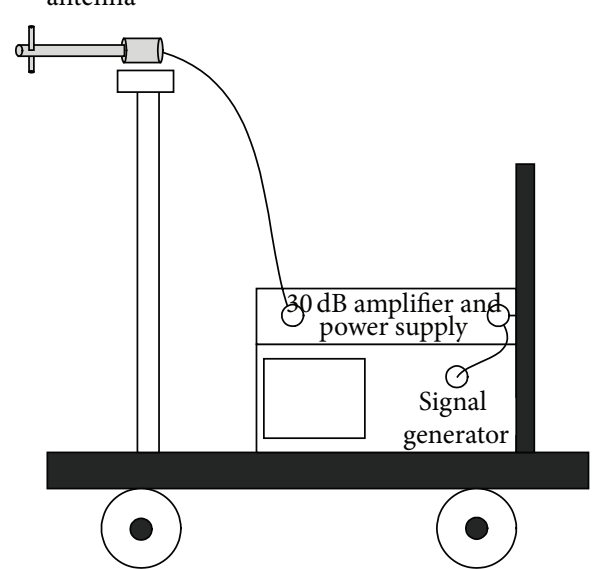

(a) Transmitter

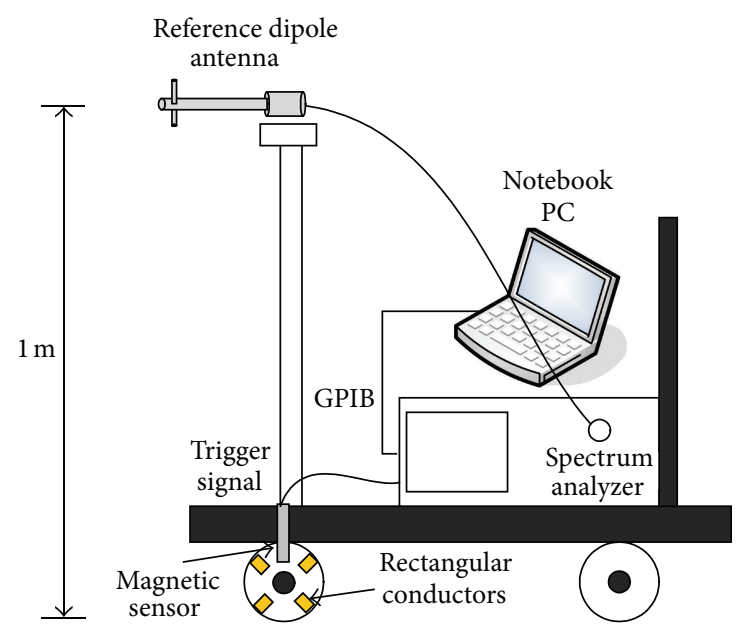

(b) Receiver

FIGURE 4: Measurement system. 


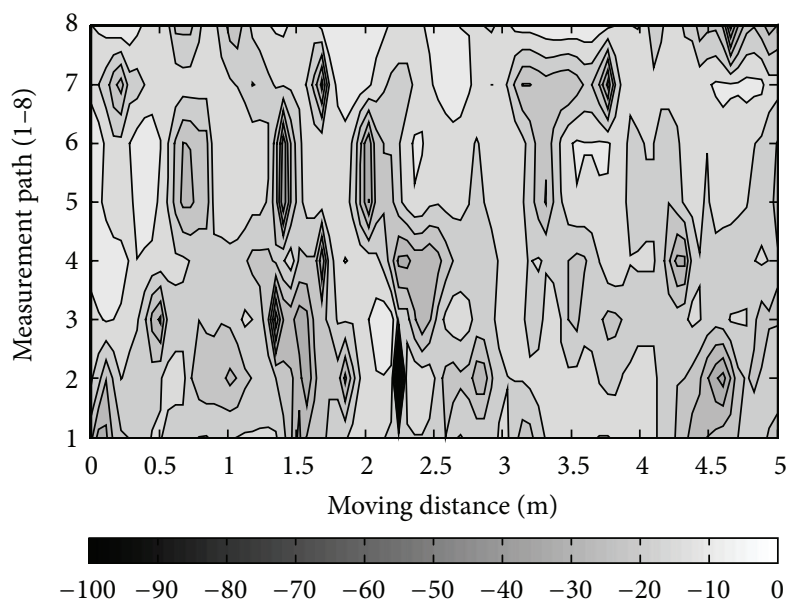

(a) $700 \mathrm{MHz}$

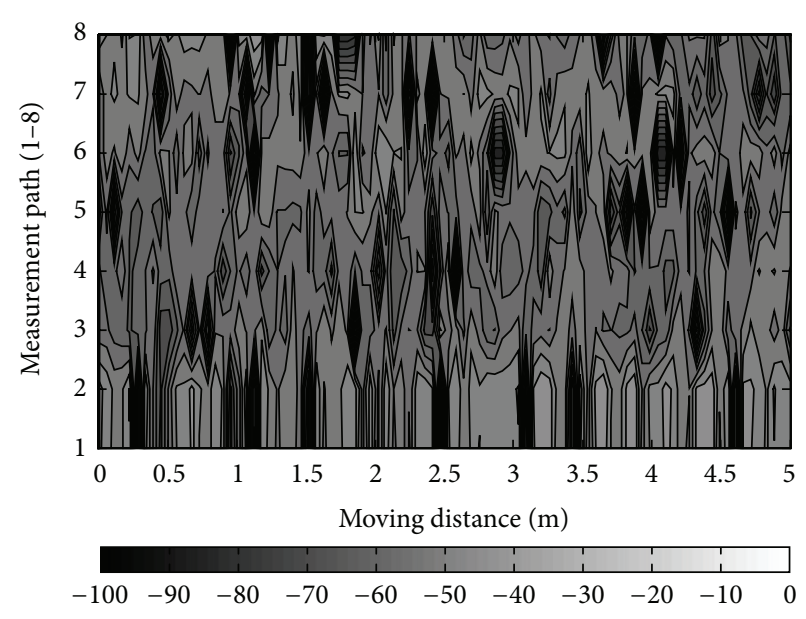

(b) $7 \mathrm{GHz}$

FIGURE 5: Measured received signal strength (fading effect).

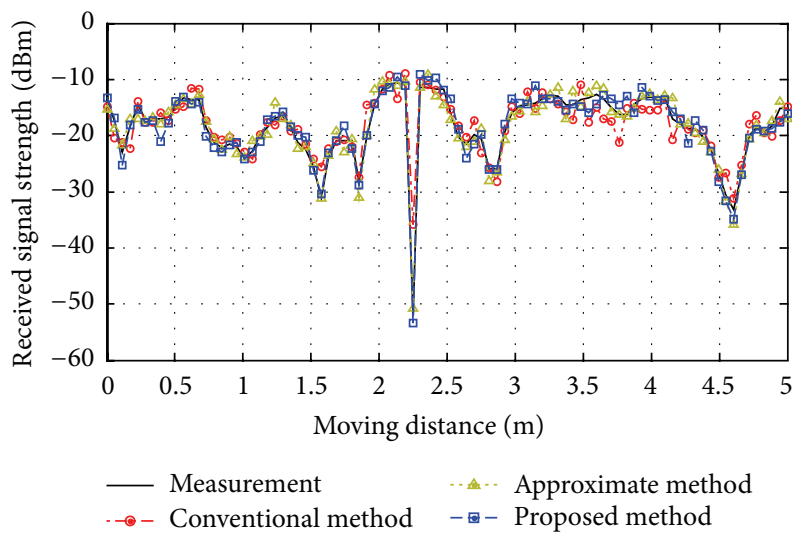

FIGURE 6: Comparison of fading effect along path 2 for measured and simulated results at $700 \mathrm{MHz}$.

each multi-path wave changes rapidly in proportion to the frequency. In other words, the prediction of the exact phase for each multi-path wave is very important, especially in high frequency bands.

Figures 6 and 7 represent comparison of fading effect along path 2 for measured and simulated results at $700 \mathrm{MHz}$ and $7 \mathrm{GHz}$, respectively. The reason that path 2 is selected for comparison is that the fading is the most severe on that path. The penetrated ray path in the simulated results using ray tracing is calculated by three methods: conventional method, approximate method, and proposed method. With the conventional method, the penetrated ray path is assumed as a straight line without refraction as shown in Figure 1. Hence, this method has the most inaccurate results. The approximate method in [12] which has some complex procedure and a more error in relation to the proposed method is compared together. At low frequency bands $(700 \mathrm{MHz})$, the simulated results with three methods are matched well with measured results in terms of fading as shown in Figure 6. However, the degree of mismatch is becoming greater as the frequency increases because the inaccurate prediction

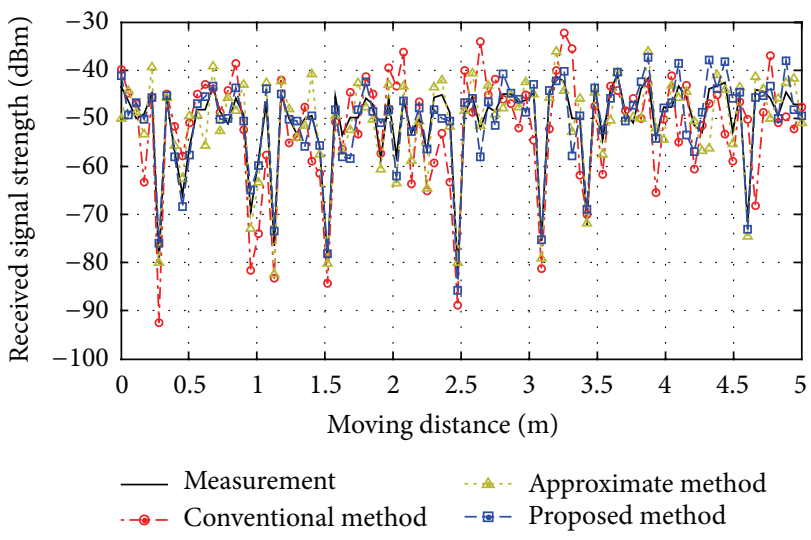

Figure 7: Comparison of fading effect along path 2 for measured and simulated results at $7 \mathrm{GHz}$.

of multipath waves including the penetrating ray path at high frequency bands due to rapid change of phase leads to an incorrect calculation when the multipath waves are added coherently. To investigate the degree of mismatch according to the methods for calculating the penetrating ray path, the root-mean-square (RMS) errors for three methods between measured results and simulated results at both frequencies are illustrated in Figure 8. With comparison between the proposed method and the conventional method, the proposed method reduces the mean RMS error by $1.38 \mathrm{~dB}$ at $700 \mathrm{MHz}$ and $4.51 \mathrm{~dB}$ at $7 \mathrm{GHz}$, respectively. This means that the conventional assumption for the penetrating ray path which is treated as a straight line without refraction is only valid at low frequency bands. On the other hand, the approximate method has more RMS errors of $0.11 \mathrm{~dB}$ at $700 \mathrm{MHz}$ and $0.89 \mathrm{~dB}$ at $7 \mathrm{GHz}$ compared with the proposed method although it is also able to improve the accuracy compared to the conventional method. Therefore the proposed method is simple to calculate because it is provided in closed-form and also can improve the accuracy of ray tracing by modeling 


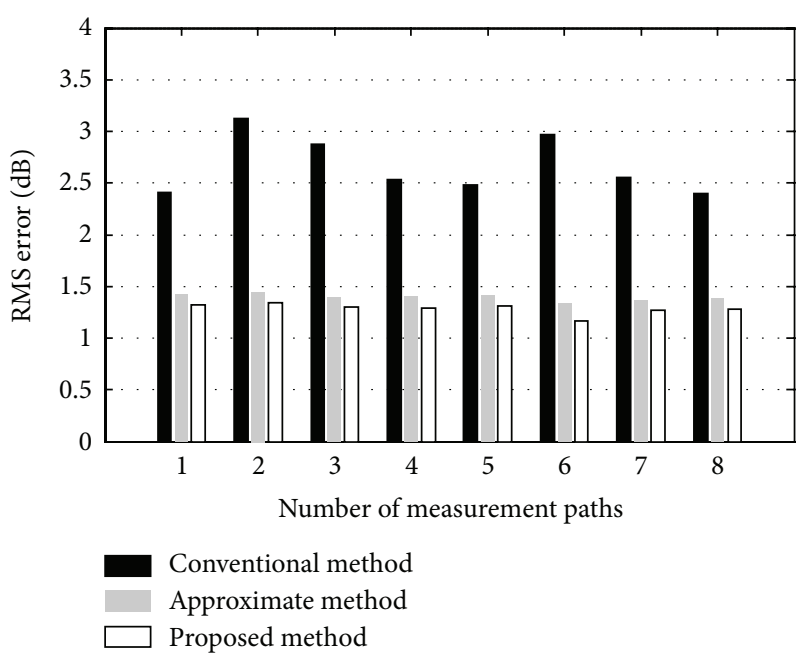

(a) $700 \mathrm{MHz}$

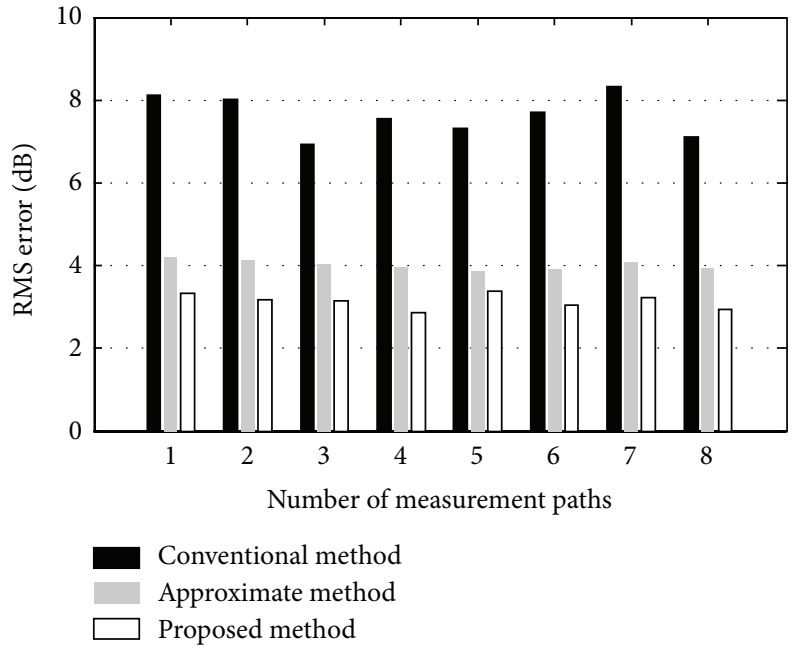

(b) $7 \mathrm{GHz}$

FIGURE 8: Comparison of RMS errors between measured results and simulated results.

the penetrating ray path exactly, especially in high frequency bands.

\section{Conclusion}

In this paper, a new method to calculate the penetrating ray path with refraction in the wall exactly and simply is proposed. For this objective, a closed-form equation is derived from Fermat's principle; hence, it has a simple form and can consider refraction when the ray penetrates through a wall. To validate the proposed method, the measured results and simulated results in terms of fading effect are compared. As a results, the significant improvement in accuracy for the proposed method is verified, especially in high frequency bands. Therefore, the proposed method is very helpful for channel analysis of indoor wireless communication using high frequency bands such as millimeter-wave WPAN application.

\section{Acknowledgment}

This research was funded by the Ministry of Science, ICT \& Future Planning MSIP, Republic of Korea, in the ICT R\&D Program 2013.

\section{References}

[1] P. Cheolhee and T. S. Rappaport, "Short-range wireless communications for next-generation networks: UWB $60 \mathrm{GHz}$ millimeter-wave wpan, and ŹigBee," IEEE Wireless Communications, vol. 14, no. 4, pp. 70-78, 2007.

[2] P. Smulders, "60GHz Radio: Prospects and Future Directions," in Proceedings of the 10th symposium on Communications and vehicular technology, pp. 1-8, Benelux, November 2003.

[3] H. Ogawa, "Millimeter-wave Wireless Personal Area Network (WPAN) and its standardization activity within IEEE 802.15," in Proceedings of the 4th International Conference on Microwave and Millimeter Wave Technology (ICMMT '04), pp. 14-17, August 2004.

[4] C.-H. Chen, C.-L. Liu, C.-C. Chiu, and T.-M. Hu, "Ultra-wide band channel calculation by SBR/Image techniques for indoor communication," Journal of Electromagnetic Waves and Applications, vol. 20, no. 1, pp. 41-51, 2006.

[5] A. Tayebi, J. Gomez, F. Saez de Adana, and O. Gutierrez, "The application of ray-tracing to mobile localization using the direction of arrival and received signal strength in multipath indoor environments," Progress in Electromagnetics Research, vol. 91, pp. 1-15, 2009.

[6] T. S. Rappaport and S. Sandhu, "Radio-wave propagation for emerging wireless personal-communication systems," IEEE Antennas and Propagation Magazine, vol. 36, no. 5, pp. 14-24, 1994.

[7] C. G. Suh, H. W. Koh, H. W. Son, and N. H. Myung, "A deterministic ray tube method for an indoor propagation prediction medel," Journal of Electromagnetic Engineering and Science, vol. 1, no. 1, pp. 48-53, 2001.

[8] S. Y. Seidel and T. S. Rappaport, "Site-specific propagation prediction for wireless in-building personal communication system design," IEEE Transactions on Vehicular Technology, vol. 43, no. 4, pp. 879-891, 1994.

[9] S.-H. Chen and S.-K. Jeng, "An SBR/image approach for radio wave propagation in indoor environments with metallic furniture," IEEE Transactions on Antennas and Propagation, vol. 45, no. 1, pp. 98-106, 1997.

[10] V. Mohtashami and A. A. Shishegar, "Efficient shooting and bouncing ray tracing using decomposition of wavefronts," IET Microwaves, Antennas and Propagation, vol. 4, no. 10, pp. 1567$1574,2010$.

[11] V. Mohtashami and A. A. Shishegar, "Inaccurate path correction in ray tracing using ray expansion method," in Proceedings of the IEEE 21st International Symposium on Personal Indoor and Mobile Radio Communications (PIMRC '10), pp. 324-328, September 2010.

[12] S. Grubisic, W. P. Carpes Jr., C. B. Lima, and P. Kuo-Peng, "Ray-tracing propagation model using image theory with a new accurate approximation for transmitted rays through walls," 
IEEE Transactions on Magnetics, vol. 42, no. 4, pp. 835-838, 2006.

[13] O. N. Stavroudis, The Mathematics of Geometrical and Physical Optics, Wiley-VCH, Weinheim, Germany, 2006. 

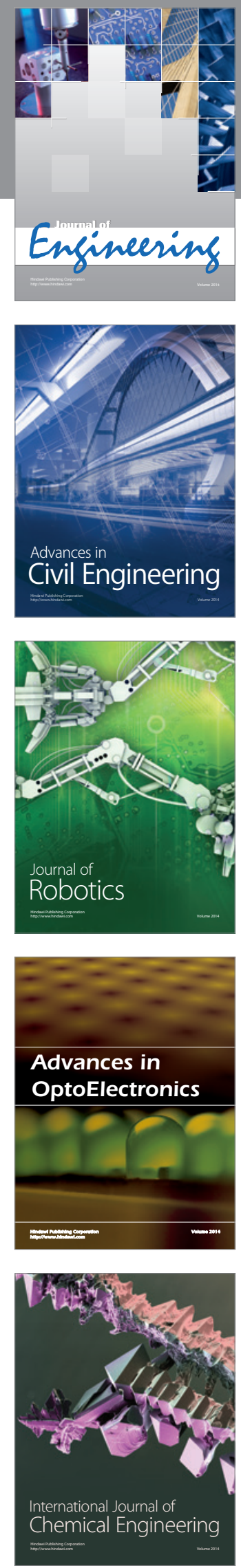

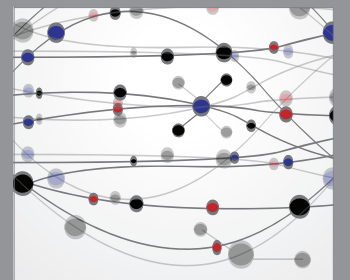

The Scientific World Journal
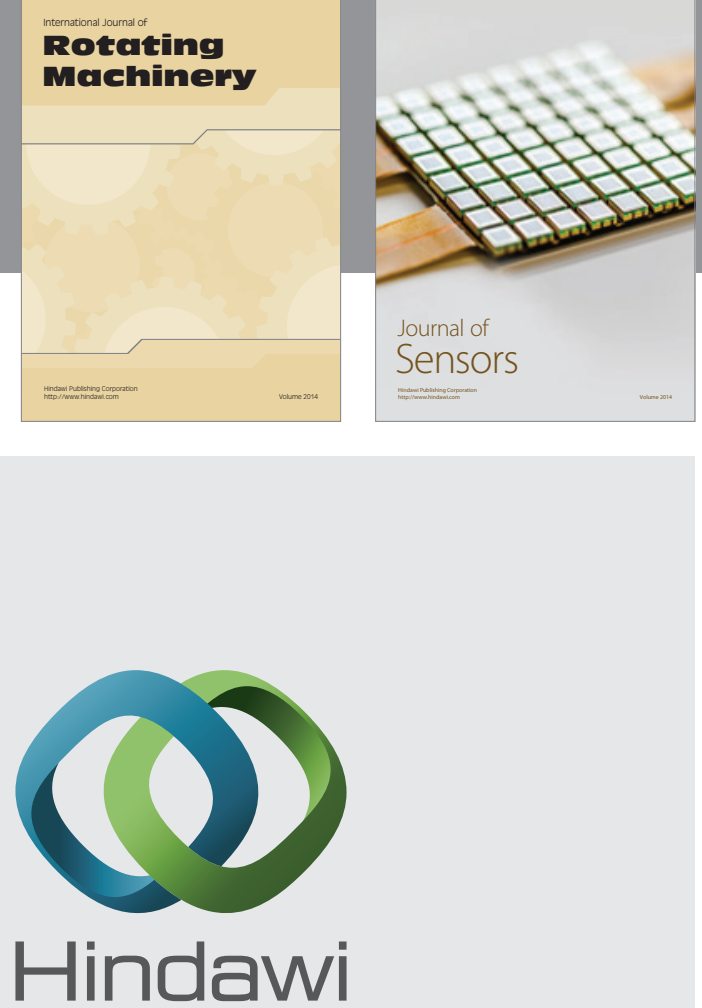

Submit your manuscripts at http://www.hindawi.com
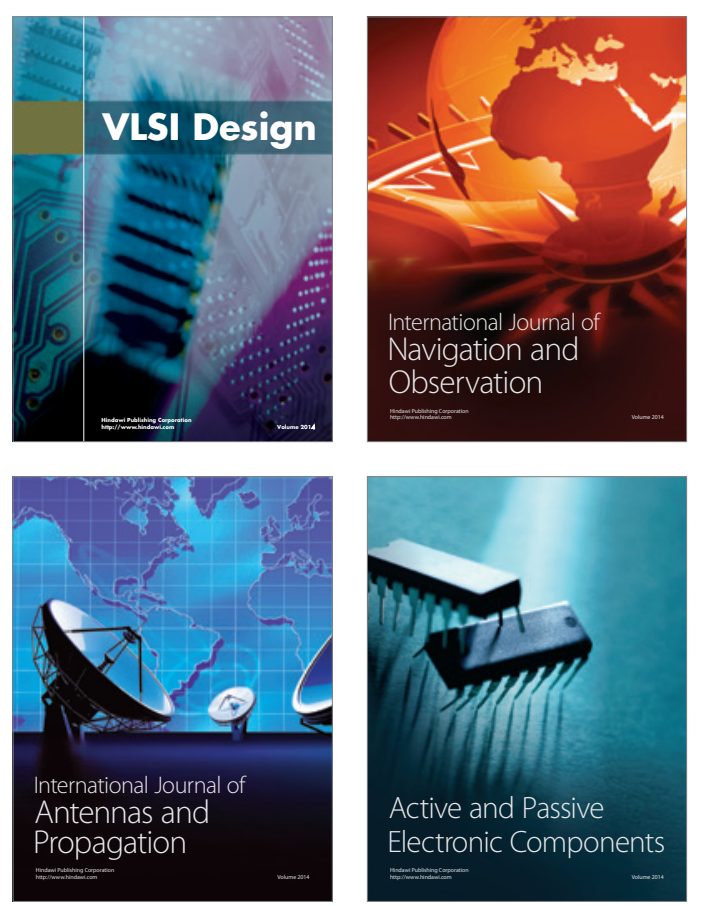
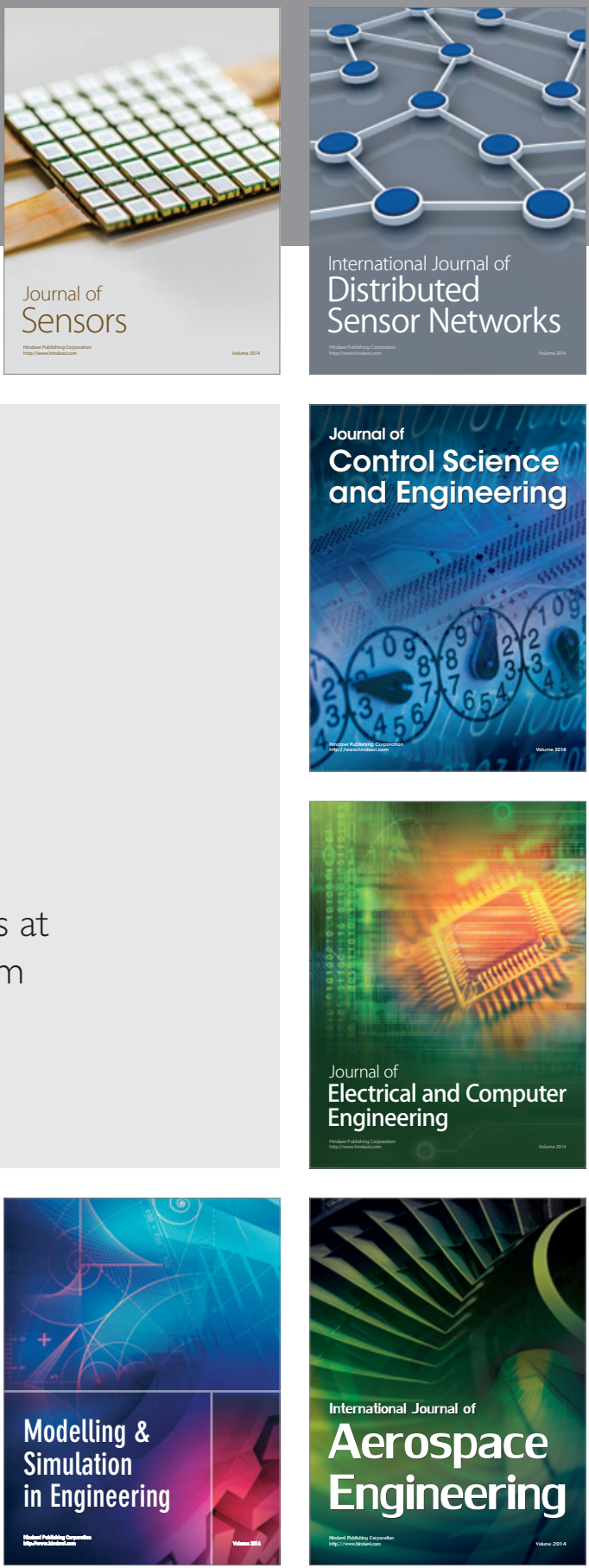

Journal of

Control Science

and Engineering
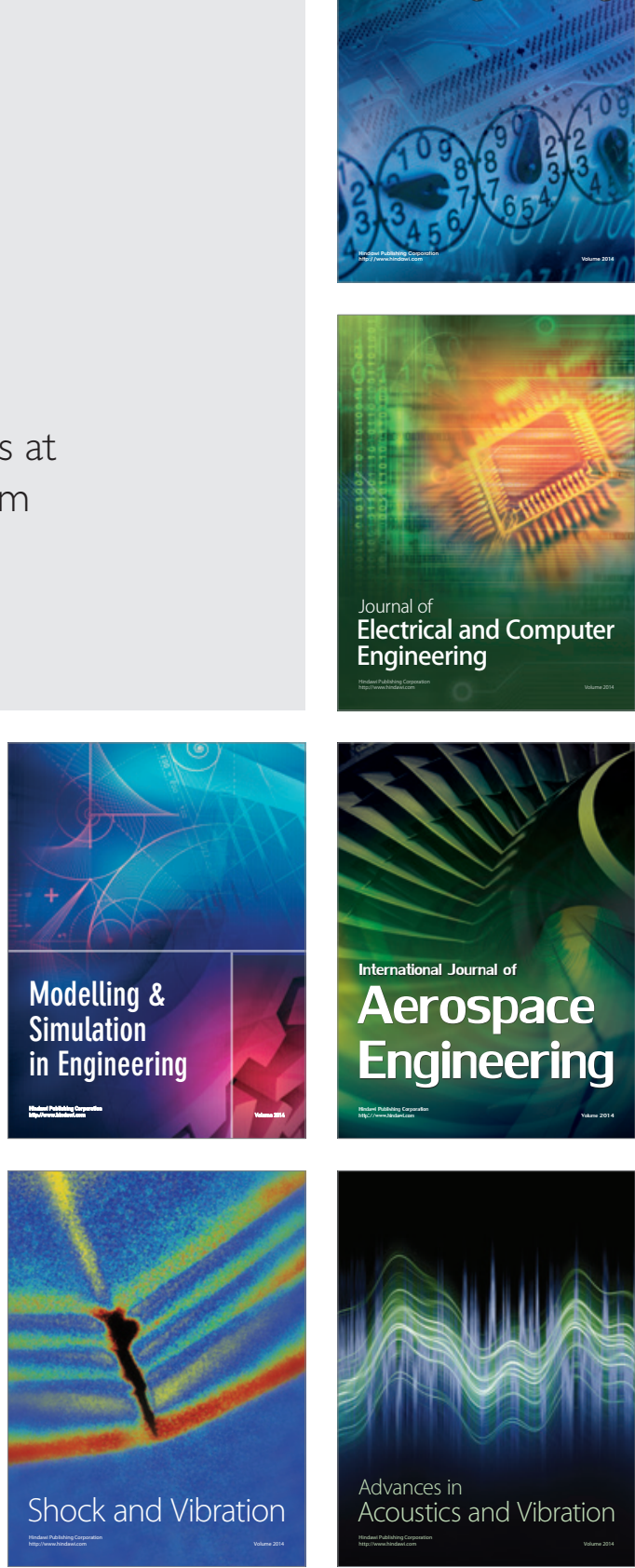